\title{
Percepção de homens sobre a influência da alimentação no câncer de próstata
}

\section{Perception of men on the influence of food on prostate cancer}

\author{
Pâmela Scarlatt D. Oliveira' • Patrícia Sousa Fernandes Queiroz • Clara Cynthia Melo Lima ${ }^{3}$ \\ Rodrigo Marques Batista Rocha ${ }^{4}$ Isabella Batista Vieira ${ }^{5}$ Henrique Andrade Barbosa ${ }^{6}$
}

\begin{abstract}
RESUMO
Objetivo: conhecer a percepção de homens sobre a influência da alimentação no desenvolvimento do câncer de próstata. Método: ensaio comunitário realizado com 60 homens cadastrados em uma Estratégia Saúde da Família da cidade de Montes Claros, com faixa etária superior a 40 anos. A coleta de dados ocorreu em três reuniões na forma de grupo focal. A análise de conteúdo temática e os dados foram organizados no software - Atlas Ti. Resultados: emergiram uma categoria e três subcategorias temáticas em que são apresentados o significado da interferência da alimentação para o câncer de próstata; a influência das interações sociais na alimentação e a importância do profissional de saúde na promoção de hábitos alimentares saudáveis.Considerações Finais: os homens entrevistados apresentam conhecimento ainda incipiente sobre a influência da alimentação na prevenção ou favorecimento do surgimento do câncer de próstata.
\end{abstract}

Palavras-chave: Câncer de próstata; Dieta; Estilo de vida.

\begin{abstract}
Objective: to know the perception of men about the influence of diet on development of prostate cancer. Methodology: a community study conducted with 60 men enrolled in a Family Health Strategy in the city of Montes Claros, aged over 40 years. Data collection took place in three focus group meetings. Thematic content analysis and data were organized in the software - Atlas TI. Results: a category and three thematic subcategories were presented in which the meaning of feeding interference for prostate cancer; the influence of social interactions on food and the importance of the health professional in promoting healthy eating habits.Final Considerations: the interviewed men present still incipient knowledge about the influence of diet on the prevention or favor of the onset of prostate cancer.

Keywords: Prostate cancer; Diet; Lifestyle.
\end{abstract}




\section{INTRODUÇÃO}

O câncer de próstata constitui hoje um problema de saúde em nível mundial, tendo aumentado sua incidência desde a década de 60 . Atualmente responde por cerca de $12 \%$ das causas de mortalidade em todo o mundo. Para o Brasil,estimamse 68.220 casos novos de câncer de próstata para cada ano do biênio 2018-2019. Em valores absolutos e considerando ambos os sexos, é o quarto tipo mais comum e o segundo mais incidente entre os homens. A taxa de mortalidade por câncer de próstata foi de 15,25 óbitos para cada 100.000 homens em 2017, diante de uma estimativa de taxa de incidência de 66,12 em 2019. Esses números confirmam que o câncer de próstata é o mais frequente no Brasil, mas não tem mortalidade alta, quando comparado com outros tipos de câncer ${ }^{(1-2)}$.

A alimentação é um dos fatores ambientais que exerce grande influência na ocorrência ou não de câncer, já que a alimentação inadequada é considerada o segundo maior fator causador de câncer que pode ser modificável ${ }^{(3)}$. Assim, a mudança no hábito alimentar da população brasileira, ocorrida nas últimas décadas, tem atraído a atenção dos órgãos reguladores e da comunidade científica como um todo, pois a substituição de alimentos in natura por alimentos processados vem contribuindo de forma contundente para - empobrecimento da dieta e aumentam as chances do desenvolvimento de cânceres (4).

A dieta rica em gordura saturada, especialmente gordura animal, e pobre em fibra aumenta o risco de câncer de próstata. Outros estudos ainda apontam $\circ$ alto consumo energético, ingestão de carne vermelha, gorduras e leite como fatores relacionados negativamente ao aparecimento do câncer. É importante destacar que a relação dieta e risco de câncer de próstata ainda está sendo estudada, buscando uma maior confirmação científica e evidenciando mais uma vez a importância de estudos nessa área ${ }^{(5)}$.

O câncer de próstata é um grave problema de saúde pública no Brasil, dessa forma é necessário um trabalho efetivo com a saúde do homem, melhorando as suas condições de saúde e colaborando para a diminuição da morbidade e mortalidade dessa população ${ }^{(6)}$. Nesse sentido, vale ressaltar a relevância da enfermagem na orientação e transmissão de informações sobre hábitos de vida saudáveis para a população masculina, por meio da realização da implementação do Processo de Enfermagem, que deve ser realizado de modo deliberado e sistemático, em todos os ambientes públicos ou privados, ocorre o cuidado desse profissional. $O$ enfermeiro não deve perder a oportunidade de abordar os homens, aproveitando as situações cotidianas da assistência, na perspectiva da promoção da saúde e detecção precoce dos cânceres, não apenas o de próstata ${ }^{(5)}$.

Portanto, considerando a relevância desse estudo para a saúde pública e o aumento de novos casos de câncer de próstata, este artigo tem como objetivo conhecer a percepção de homens sobre a influência da alimentação no desenvolvimento do câncer de próstata.

\section{MÉTODO}

Trata-se de um estudo de abordagem qualitativa, baseado no método de ensaio comunitário, que se traduz em uma pesquisa que visa à implementação ou avaliação de intervenções dirigidas à prevenção primária por meio da modificação de fatores de risco em uma população bem definida.

Todos os homens com faixa etária superior a 40 anos e sem déficits cognitivos que compareceram a unidade de saúde no mês da coleta de dados foram convidados a participar do primeiro grupo focal, que foi realizado na última semana do mês de novembro. Os integrantes da pesquisa que compareceram à primeira reunião foram 60 homens cadastrados em uma Estratégia de Saúde da Família (ESF) da cidade de Montes Claros, Minas Gerais, Brasil, sendo que tal recomendação referente a idade se apoiou nas diretrizes da Sociedade Brasileira de Urologia que determina, até então, imperativa a realização do exame digital da próstata anualmente nessa faixa etária ${ }^{(7)}$. Na segunda reunião compareceram 32 homens e na terceira 25 indivíduos.

A coleta dos dados ocorreu em três reuniões na forma de grupo focal, em que foram realizadas algumas perguntas norteadoras: "Você acha que a alimentação influencia no aparecimento do câncer?"; "Fale sobre suas experiências positivas e negativas em relação à alimentação e proteção contra aparecimento de cânceres"; "Fale sobre seu conhecimento e a relação da sua alimentação e o aparecimento de doenças como o câncer de próstata”. As reuniões tiveram duração média de uma hora, e aconteceram no período noturno quando os homens não estavam em horário de trabalho, o que justifica a boa adesão dos mesmos. Foram utilizadas câmeras digitais para gravar as imagens do grupo. As falas foram gravadas com gravador eletrônico e transcritas de modo a permitir uma melhor análise das mesmas.

Os sujeitos foram identificados pela letra $E$, seguida do número da entrevista, para garantir o anonimato. Para a análise dos dados qualitativos, foi realizada a análise de conteúdo temática e os dados foram organizados no software - Atlas Ti (Qualitative Research and Solutions), que auxiliou no primeiro nível de análise e na codificação dos momentos significativos evidenciados nos dados.

Utilizou-se os seguintes passos para a análise das falas: ordenação dos dados (transcrição das gravações; releitura do material; organização dos relatos); classificação dos dados realizada no software (leitura dos textos; constituição de um corpus de comunicações; leitura transversal de cada corpo com o recorte de "unidades registro"; classificação por temas mais relevantes) e análise final do material gerado no Atlas Ti (considerando os objetivos do trabalho, o referencial teórico e os temas que emergiram dos depoimentos dos clientes), sendo agrupadas as falas por convergência ${ }^{(8)}$. Os resultados foram apresentados em folders para os profissionais da ESF e para os participantes da pesquisa.

O estudo atendeu à Resolução $N^{\circ} 466 / 2012$ e foi aprovado pelo Comitê de Ética em Pesquisa com o número de protocolo do CEP/SOEBRAS: $403.501 / 2013$. Todos os participantes assinaram um termo de consentimento livre e esclarecido.

\section{RESULTADOS}

A grande maioria dos entrevistados possuía idade entre 50 e 55 anos, era casado e tinha menos de nove anos de escolaridade. Destaca-se que maioria significativa já havia realizado algum dos exames preventivos para o câncer de próstata, como toque retal ou PSA, pelo menos uma vez.

As falas foram reunidas no software Atlas Ti e foi observado que alguns verbos e substantivos se repetiram com uma maior frequência, conforme pode ser evidenciado no Quadro I: 
Quadro I -Verbos e substantivos que mais se repetiram nas falas dos participantes.

\begin{tabular}{|c|c|}
\hline Verbos & Substantivos \\
\hline $\begin{array}{c}\text { Fazer, comer, cozinhar, fritar, conhecer, } \\
\text { prevenir, fumar, consultar, achava, beber, } \\
\text { mudar. }\end{array}$ & $\begin{array}{c}\text { Próstata, alimentação, carne, gordura, } \\
\text { hábitos, prevenção, esposa, amigo, gosto- } \\
\text { so, frito, cerveja, verdura, feijão, cigarro, } \\
\text { médico. }\end{array}$ \\
\hline
\end{tabular}

Após a análise minuciosa das falas dos participantes do estudo, emergiram 3 categorias que serão apresentadas a seguir:

\section{O significado da interferência da alimentação para} o câncer de próstata

Os homens foram questionados sobre a sua percepção quanto aos hábitos alimentares e o surgimento do câncer. Percebe-se que a maioria dos participantes do estudo possuem algum conhecimento sobre a alimentação como ferramenta de proteção ao câncer, entretanto, como pode ser observado nas falas a seguir, esse conhecimento não é suficiente para favorecer a predileção por alimentos saudáveis de modo efetivo na rotina alimentar.

"A questão da comida da pessoa que alimenta de muita carne gordurosa, no caso é questão de educação alimentar..."(EI)

"O problema é que esses alimentos, os mais gostosos, são esses mesmos que causam mal mesmo, mas eu já me acostumei com eles." (E2)

"Mais eu não como pedaço de gordura assim todo dia, só de vez em quando..." (E4) (E8)

"Os enlatados são os perigosos né, mas eu ainda como".

"Olha só pra você ver o que eu fiz ontem... Ontem eu comi peixe frito, num estava muito correto, né, por ser frito... depois tomei um caldo de peixe, num ta bom? ... só que hoje eu comi uma feijoada". (EIO)

Dúvidas como a relação da obesidade e o surgimento de câncer também emergiriam nos grupos focais, como pode ser observada na fala E6: "tem alguma coisa a ver, a pessoa ser gordo e dar o câncer?". Tais questionamentos reforçaram a necessidade de fornecer orientações sobre alimentação saudável nas reuniões como os homens. Após essas orientações, os participantes foram questionados sobre a possibilidade de mudança dos hábitos alimentares. Alguns dos entrevistados, mesmo de posse de informações relativas ao assunto, persistem na adoção de hábitos considerados nocivos para a saúde geral, outros, porém, reconhecem, como base em seu histórico alimentar, comportamentos que podem ser modificados, como pode ser observado nas falas a seguir:

"Tem que comer bem que se quiser viver melhor, evitar esses trem que faz mal...”(E2)

"Agora tenho que sair daqui não fumando, é deixar mesmo o cigarro e a gordura...”(E8)

"Depois do que você disse aí, eu vou escolher aqueles lá mesmo que é bom, mas em algum tempinho eu vou comer um pouquinho de gordura também. (risos)”. (EIO)

A influência das interações sociais na alimentação

O apoio recebido pelos homens é um fator que lhes dá sustentação para que não desistam de se cuidar, em que a família, amigos e os profissionais de saúde têm a capacidade de exercer influências positivas ou negativas no padrão alimentar dos homens. A família é uma forte influenciadora no quesito alimentação, principalmente quando se trata da dieta masculina que, na maioria das vezes, é providenciada por outra pessoa.

"Esses dias a feijoada que minha mulher fez, eu gostei demais, era só verdura com feijão, você já viu? Ficou boa”. (E2)

"A verdura agora que eu tô alimentando que eu como agora mesmo, num é cozida no óleo, minha mulher só cozida os pedaços grandes, cozinha, é muito mais gostosa, num leva gordura não... só ferventada”. (E8)

"Final de semana quando junta todo mundo em casa a comida sempre tem salada, carne assada ou um franguinho ensopado". (E/3)

"Eu só como em casa com a família no final de semana, porque trabalho lá na firma os outros dias e não dá tempo de ir em casa". (E8)

"Se eu comesse com minha esposa com certeza seria mais saudável, mas não dá tempo de almoçar em casa todo dia”. (E6)

"Final de semana depois da partida de bola com os amigos sempre tem aquela cervejinha, feijoada". (EIO)

"Fica sem graça sair com os amigos pro buteco e não comer as coisas que tem lá”. (E8)

"Eu não tenho muitos amigos com vida saudável não". (E7)

\section{O papel do profissional de saúde na promoção de} hábitos alimentares saudáveis

A subcategoria em questão coloca em relevo o papel do profissional de saúde no que concerne à orientação de hábitos alimentares saudáveis. As consultas devem ser um espaço fecundo para orientações que favoreçam a compreensão do indivíduo acerca do seu protagonismo frente a sua condição de saúde e a importância da adoção de hábitos de vida saudável, como a alimentação balanceada, a fim de minimizar os fatores de risco que estão envolvidos no desenvolvimento de diferentes doenças, como, por exemplo, o câncer. Nas falas abaixo, percebe-se tanto a importância, como a deficiência de informações oferecidas pelos profissionais de saúde:

"O médico me avisou que eu tenho que melhorar a alimentação mesmo, mas ninguém me falou sobre esses outros alimentos diferentes não".

"Eu sei que não pode comer muito sal nem açúcar porque a enfermeira falou isso comigo o dia que foi visitar 
minha mãe”. (E5)

"É os médicos mandam a gente tomar o vinho, falam que é bom pra saúde”. (E6)

\section{DISCUSSÃO}

$\mathrm{Na}$ análise dos verbos e substantivos que mais se repetiram, houve uma preponderância dos verbos "comer, cozinhar, fritar" e dos substantivos "próstata e alimentação" se justifica pela natureza da abordagem deste estudo: a percepção sobre a relação da alimentação e sua influência no câncer de próstata. Nota-se que prevenção/prevenir foram bastante mencionados, o que pode denotar uma percepção do papel da alimentação no surgimento do câncer de próstata.

Os homens participantes do estudo demonstram conhecimento parcial das influências negativas de alimentos com alto teor de gordura sobre a saúde. Autores ${ }^{(8)}$ enfatizam a importância de evitar o álcool, gorduras e carnes em geral, especialmente carne vermelha, uma vez que o câncer necessita de calorias para se desenvolver. Destacam ainda que a melhor forma para uma alimentação saudável é comer muita verdura e vegetais, grãos e legumes, produtos de soja, chá verde ou branco, ingerir cerca de dois litros de água por dia, pelo menos 25 gramas de fibras, além de vitaminas C, D e E e o mineral selênio.

Estudo transversal com $21 \mathrm{l}$ idosos apresentando diag $\neg$ nóstico prévio de câncer de próstata observou uma alta inadequação de consumo de frutas e hortaliças entre os pacientes estudados, tanto em frequência quanto em quantidade, com uma média de consumo abaixo da recomendação da Organização Mundial da Saúde (OMS). Os autores consideraram os resultados preocupantes, uma vez que o consumo de frutas e verduras não está relacionado apenas à prevenção do câncer, mas também ao controle dessa doença ${ }^{(9)}$.

O aparecimento constante de palavras como "cigarro/ fumar", "cerveja/beber", "carne/gordura", "frito/fritar" pode indicar o conhecimento da influência do cigarro e do álcool no surgimento do câncer, bem como sinalizar que o discurso da adoção à hábitos de vida saudável, tão difundido pelas mídias e pelas políticas de saúde, consegue chegar ao público alvo. Entretanto, isso não garante a verdadeira adoção desses hábitos, pois a grande maioria dos entrevistados referiu ainda ser resistente a mudanças nos hábitos alimentares e estilo de vida saudável. Embora exista uma ampla discussão sobre a masculinidade, ainda há insuficiência de estudos sobre o empenho masculino voltado para o estilo de vida saudável e a promoção da saúde ${ }^{(10)}$.

No que concerne à mudança na perspectiva da alimentação saudável e protetora, notou-se uma persistência de comportamentos inadequados, mesmo após serem transmitidas informações sobre o assunto, o que demonstra a necessidade de abordar mais frequentemente 0 tema alimentação durante as consultas e a importância de se investigar novas estratégias de prevenção, promoção da saúde e transmissão de conhecimento entre os homens ${ }^{(10)}$. Tais estratégias devem englobar a família, considerada unidade primária de cuidado e um espaço de interação, troca de informações e apoio mútuo, além de influenciar significativamente tanto nas modificações de hábitos de vida quanto na busca de soluções no eventual surgimento de um problema de saúde (II).

Sobre isso, autores afirmam que devido as questões culturais, comportamentais e ao próprio desconhecimento, a população masculina, em sua maioria, tende a protelar a procura por assistência à saúde. Para além disso, estudo demonstra que os homens apelam diretamente para a saúde curativa, utilizando os serviços de saúde em momentos de doença ou em casos de urgência e, exatamente por isso, tornam-se mais suscetíveis a morbidades e agravos (12).

Por meio das falas dos homens, percebe-se que existe uma resistência dos amigos em ter mudança nos hábitos de vida e alimentação, sendo que isso se torna um fator dificultador para os homens que querem ter uma alimentação saudável, visto que não encontram apoio e deixam de ser bem aceitos em seu grupo social. É necessário que sejam investigadas novas maneiras de abordar os homens em grupos incentivando mudanças na alimentação.Ainda são escassos estudos avaliando as interações entre a alimentação do homem e seus grupos sociais, em especial suas amizades e companheiros de saídas (12).

É válido acrescentar ainda que a SAE se constitui no planejamento das ações de enfermagem para solucionar os problemas dos pacientes e promover o cuidado individualizado durante as consultas de enfermagem. Dentre as etapas da SAE, destaca-se o Diagnóstico de Enfermagem, definido como características necessárias para diagnosticar as respostas humanas a determinados problemas de saúde. A taxonomia de diagnósticos de enfermagem, reconhecida oficialmente no mundo e mais difundida no Brasil, é a North American Nursing Diagnosis Association - NANDA Internacional (13-15).

No caso dos pacientes como os desse estudo que se alimentam de forma inadequada, os principais diagnósticos de enfermagem que podem ser empregados são: "Nutrição desequilibrada: mais do que as necessidades corporais" que consiste na ingestão de nutrientes que excedem às necessidades metabólicas, tendo como características definidoras:"comer em resposta a estímulos externos";"comer em resposta a estímulos internos que não a fome"; "estilo de vida sedentário"; "padrão de alimentação disfuncional”; "peso $20 \%$ acima do ideal para altura e compleição". Existem ainda, fatores relacionados:"ingestão excessiva em relação à atividade física" e "ingestão excessiva em relação às necessidades metabólicas". Outros possíveis diagnósticos são "Ingestão de líquidos diminuída" e "Conhecimento deficiente" (13).

O(a) enfermeiro (a), sobretudo na Atenção Básica, pode contribuir de modo efetivo na prevenção do câncer de próstata através de atividades educativas e informativas à comunidade, incluindo escolas e universidades, centrando-se na promoção de saúde e qualidade de vida; realização de ações preventivas, através da busca ativa, visando rastrear novos casos dessa neoplasia, através de visitas domiciliares; manter os homens informados sobre os fatores de riscos; promover orientação sobre a importância da realização dos exames preventivos, oferecer apoio para elaborar e atualizar condutas a serem seguidas; realizar grupo de homens, cujo tema seja saúde do homem; identificar fatores de riscos através de abordagens em linguagem clara e objetiva ${ }^{(6)}$.

Além dessas, outras intervenções também podem ser realizadas, com base nos diagnósticos de enfermagem elaborados, como a medida do peso e estatura corpórea na avaliação do estado de saúde dos pacientes e no acolhimento de medidas preventivas e terapêuticas, no que antecede o combate ao sobrepeso e obesidade. Os enfermeiros devem incentivar a adoção de mudanças para promover um estilo de 
vida mais saudável, incluindo auxiliar os homens a superar os obstáculos relacionados com modificações na dieta e a prática de exercícios físicos regulares ${ }^{(15)}$.

\section{CONSIDERAÇÕES FINAIS}

Diante do conhecimento ainda incipiente sobre a influência da alimentação como fonte de proteção ou de favorecimento do surgimento do câncer de próstata por parte dos homens entrevistados, torna-se fulcral que os profissionais de enfermagem e os demais profissionais da saúde, nos diferentes espaços de cuidado, orientem a população masculina sobre hábitos alimentares saudáveis, focando nos alimentos que previnem o aparecimento do câncer, além de acompanharem os resultados de estudos que apresentem melhores níveis de evidência em termos de prevenção do câncer de próstata e fatores de risco, o que também poderá oferecer subsídios para a elaboração e atualização de protocolos e condutas a serem seguidos.

\section{REFERÊNCIAS}

1. Ministério da Saúde. Instituto Nacional de Câncer. Estimativa 2018: incidência de câncer no Brasil. Rio de Janeiro (RJ): INCA; 20I7. Disponível em: http://wwwl. inca.gov.br/estimativa/2018/estimativa-2018.pdf

2. Ministério da Saúde. Instituto Nacional de Câncer. Medida mais eficaz para prevenção de câncer no homem é a adoção de hábitos saudáveis. 2019. Disponível em: https://www.inca.gov.br/imprensa/ medida-mais-eficaz-para-prevencao-de-cancer-nohomem-e-adocao-de-habitos-saudaveis

3. Nemer BC, Carvalho MSB. A dieta vegetariana na prevenção do câncer: uma revisão de literatura. Revista Ciências Nutricionais Online [Internet]. 2019 [acesso em 7 jul 2019]; 3 (I): 5-I3. Disponível em: http://unifafibe.com.br/ revistasonline/arquivos/cienciasnutricionaisonline/ sumario/82/03062019134853.pdf

4. Santos PS, Lourival NBS. Consumo de compostos químicos oriundos de embutidos e sua correlação com o desenvolvimento do câncer: uma revisão. Rev. Terra \& Cult. [Internet]. 2019 [acesso em 07 jul 20l9]; 34 (67): 73-83. Disponível em: http://periodicos.unifil.br/ index.php/Revistateste/article/view/970/907

5. Czorny RCN et al. Fatores de risco para o câncer de próstata: população de uma unidade básica de saúde. Cogitare enferm [Internet]. 2017 [acesso em 13 set 2019]; v. 22(4). Disponível em: http://www.saude. ufpr.br/portal/revistacogitare/wp-content/uploads/ sites/28/2018/08/5 I823-2I7936-I-PB.pdf

6. Vasconcelos $\mathrm{LI}$ et al. Atuação do enfermeiro na prevenção do câncer de próstata: revisão integrativa. Rev. Bra. Edu. Saúde [Internet]. 2019 [acesso em 07 jul 2019]; 9 (2): 2I-26. Disponível em: https:// www.gvaa.com.br/revista/index.php/REBES/article/ view/6384/5589
7. Minayo MCS. O desafio do conhecimento: pesquisa qualitativa em saúde. I2 . ed. São Paulo: Hucitec; 2012.

8. Ramos SS et al. Conhecimentos, mitos e implicações para o cuidado de enfermagem no câncer de mama masculino. Revista Enfermagem Atual [Internet]. 2017 [acesso em: 20 out 2019]; 83(21): 67-72. Disponível em: https://revistaenfermagematual.com.br/index.php/ revista/article/view/287//78

9. Cordeiro MD et al. Fatores associados ao consumo de frutas e hortaliças por idosos portadores de câncer de próstata. BRASPEN J [Internet]. 2017 [acesso em 07 jul 2019]; 32 (2): I08-13 Disponível em: http://www.braspen.com.br/home/wp-content/ uploads/20 I 7/08/02-AO-Fatores-associados-aoconsumo-de-frutas.pdf

10. Debruyne FMJ et al. Testosterone treatment is not associated with increased risk of prostate cancer or worsening of lower urinary tract symptoms: prostate health outcomes in the Registry of Hypogonadism in Men. BJU international [Internet]. 2017 [acesso em 05 jul 2019]; I I 9(2):2 I 6-224. Disponível em: https://www. ncbi.nlm.nih.gov/pubmed/27409523

11. Mathias $C V$ et al. $O$ adoecimento de adultos por câncer e a repercussão na família: uma revisão da literatura. Rev. de Atenção à Saúde [Internet]. 2015 [acesso em 06/I2/2019]; I3(45):80-86. Disponível em: file:///C:/ Users/patri/Downloads/oadoecimentoporcncer\%20 (4).pdf

12. Oliveira BMS et al. Hábitos alimentares relacionados ao desenvolvimento de doenças cardiovasculares em adolescentes. Uniciências [Internet]. 2015 [acesso em I3 set 2019]; 19(I): 55-6I. Disponível em: http://www. unirio.br/cecane/arquivos/3 |56 I | 938IPB.pdf

13. Nanda. Diagnósticos de enfermagem da NANDA: definições e classificação 2018-2020. II a Ed. Porto Alegre:Artmed; 2018.

14. Ferreira $M A$ et al. Prevalência do diagnóstico de enfermagem nutrição desequilibrada em hipertensos. Cogitar enferm [Internet]. 2016 [acesso em 13 set. 2019]; v. 2 I (3): 0I-09. Disponível em: https://revistas. ufpr.br/cogitare/article/view/48942/29419

15. Oliveira APS, Santos WL. O conhecimento do enfermeiro sobre a obesidade-revisão de literatura. Rev. Cient. Sena Aires [Internet]. 2018 [acesso em I5 set. 20I9]; v. 7(2): |4|-|47. Disponível em: http:// revistafacesa.senaaires.com.br/index.php/revisa/ article/view/3 |4/224 